\title{
Isolation, Characterization, and Evolutionary Divergence of Mouse RNase 6: Evidence for Unusual Evolution in Rodents
}

\author{
Kimberly D. Dyer, ${ }^{1}$ Helene F. Rosenberg, ${ }^{1}$ Jianzhi Zhang ${ }^{2}$ \\ ${ }^{1}$ Eosinophil Biology Section, Laboratory of Allergic Diseases, National Institute of Allergy and Infectious Diseases, \\ National Institutes of Health, Bethesda, MD 20892, USA \\ ${ }^{2}$ Department of Ecology and Evolutionary Biology, University of Michigan, Ann Arbor MI 48109, USA
}

Received: 26 February 2004 / Accepted: 7 June 2004 [Reviewing Editor: Dr. Lauren Ancel Meyers]

Abstract. The evolution of the ribonuclease A (RNase A) vertebrate-specific enzyme family is interesting in that specific gene lineages appear to be responding to unique selective pressures in wildly diverse manners to generate proteins that are capable of reducing the infectivity of viruses, killing systemic pathogens, and inducing the growth of blood vessels all while maintaining the signature motifs of a ribonuclease. In this paper, we present the DNA sequence and gene structure of Mus musculus RNase 6 and examine the expression pattern and enzymatic activity of the recombinant protein. M. musculus RNase 6 has a limited expression pattern compared to human RNase 6 and is an efficient ribonuclease, with a catalytic efficiency 17 -fold higher than that of human protein. Evolutionary analysis reveals that RNase 6 was subject to unusual evolutionary forces $\left(d_{\mathrm{N}} / d_{\mathrm{S}}=1.2\right)$ in an ancestral rodent lineage before the separation of $M u s$ and Rattus. However, more recent evolution of rodent RNase 6 has been relatively conserved, with an average $d_{\mathrm{N}} / d_{\mathrm{S}}$ of 0.66 . These data suggest that the ancestral rodent RNase 6 was subject to accelerated evolution, resulting in the conserved modern gene, which most likely plays an important role in mouse physiology.

The GenBank accession numbers for the new genes presented here are as follows: Mus musculus, AY545655; Rattus norvegicus, AY545654; Mus spicilegus, AY545653; Mus caroli, AY545651; and Mus pahari, AY545652.

Correspondence to: Kimberly D. Dyer; email: kdyer@niaid.nih.gov
Key words: Ribonuclease - RNase A superfamily - RNase k6 - RNase 6 - Host defense

\section{Introduction}

Ribonuclease 6 is a member of the ribonuclease A (RNase A) superfamily, which is believed to be the only enzyme family that is limited to vertebrates (Lander et al. 2001). This family has been well characterized structurally, with most members having eight cysteines as well as the lysine and the two histidines that form the catalytic triad (Beintema et al. 1988; Rosenberg et al. 1995). However, this family of ribonucleases is functionally quite diverse and not particularly well understood. There are several distinct lineages within the larger RNase A superfamily (Fig. 1). Pancreatic ribonucleases (RNase 1) have been isolated from a broad spectrum of mammalian species (Dubois et al. 2003; Zhang et al. 2002b); they are expressed in a number of tissues including the pancreas (Futami et al. 1997) and serve primarily as digestive enzymes. Ribonuclease 4 (RNase 4) is most similar to pancreatic ribonuclease and is also expressed in many human tissues (Rosenberg and Dyer 1995). Angiogenin (RNase 5) is structurally distinct in that it has only six of the canonical eight cysteines. It was originally identified as a factor stimulating blood vessel growth (Strydom 1998). Most recently, mouse and human angiogenins have been identified as microbicidal agents against both bacteria and fungi, suggesting that angiogenins con- 




Fig. 1. The ribonuclease A (RNase A) superfamily. The neighborjoining phylogenetic tree of the human (h) and mouse (m) RNase A ribonucleases. Swiss protein database accession numbers for amino acid sequence are as follows. Ears (due to space constraints, only 4 of the 10 or more mouse Ears are shown here): hRNase 2/EDN, P10153; hRNase 3/ECP, P12724; Ear 1, P97426; mEar 2, P97425; mEar 5, O35292; mEar 4, AF017259; and mEar 11, AYO15178. Pancreatic ribonucleases: hRNase 1, P07998; mRNase 1, P00683. RNase 4: hRNase 4, P34096; and mRNase 4, Q9JJH1. Angiogenins: hRNase 5, P03950; mRNase 5/Ang, P21570; mAngrp/Ang2, Q64438; mAnglp/Anrp2/Ang3, P97802; and mAng4, AY219870. Ear-like: hRNase 6, Q93091; mRNase 6, Q9D244; hRNase 7, Q9H1E1; and hRNase 8, Q8TDE3. Tree and bootstrap percentages (from 2000 replications, at branching points) were obtained using MEGA2 (Kumar et al. 2001).

tribute to innate host defenses (Hooper et al. 2003). Similarly, human EDN (RNase 2) and ECP (RNase 3 ), initially characterized as eosinophil cationic toxins, exhibit antiviral and antibacterial activities and are thought to play a role in eosinophil-mediated host defense (Domachowske et al. 1998a, b; Rosenberg and Domachowske 2001).

The evolution of this vertebrate-specific enzyme family is interesting in that specific lineages appear to be responding to unique selective pressures in wildly diverse manners. Human EDN (RNase 2) and ECP (RNase 3) remain among one of the most rapidly evolving genes known in primates (Rosenberg et al. 1995) and the orthologous mouse genes, mouse eosinophil-associated ribonucleases (Ears), have undergone rapid and divergent evolution to produce as many as 10-15 paralogous genes (Batten et al. 1997; Cormier et al. 2001; Larson et al. 1996; McDevitt et al. 2001; Singhania et al. 1999; Zhang et al. 2000). The pancreatic ribonuclease lineage has undergone at least two species-specific duplications, one in ruminants, resulting in pancreatic, seminal, and brain ribonucleases (Beintema et al. 2003; Sasso et al. 1999), and another in the leaf-eating monkey douc langur, resulting in two distinct enzymes with differing substrate specificities and optimal pHs (Zhang et al. 2002b). Ribonuclease 8 has been deactivated in a number of species (Zhang et al. 2002a), and interestingly, the orthologue of human RNase 8 and that of a closely related gene, RNase 7 (Zhang et al. 2003), have not been discovered in either the mouse or the rat genome.

DNA sequences encoding part of RNase 6 have been isolated from a variety of species (Deming et al. 1998; Pietrowski and Forster 2000; Rosenberg and Dyer 1996). Although the RNase 6 gene lineage is most closely related to the EDN/ECP (RNase 2/3) lineage (Fig. 1), RNase 6 genes examined thus far appear to be responding to more conservative evolutionary constraints. In this paper, we present the DNA sequence and gene structure of Mus musculus RNase 6 (mRNase 6) and examine the expression pattern and enzymatic activity in this species. In addition, we sequenced the RNase 6 gene from four other rodent species-Mus pahari, Mus caroli, Mus spicilegus, and Rattus norvegicus. Our analyses suggest that this gene was subject to unusual evolutionary forces in an ancestral rodent lineage and that RNase 6 likely has altered functions among rodents.

\section{Materials and Methods}

\section{PCR Amplification of RNase 6 from Rodent Species}

Genomic DNA from Mus pahari (shrew mouse) and Mus caroli (Ryukyu mouse) was provided by Anthony Furano (National Institutes of Health, Bethesda, MD) and Mus spicilegus (steppe mouse) genomic DNA was purchased from Jackson Laboratories. Mus musculus (house mouse) and Rattus norvegicus (Norway rat) genomic DNA was isolated from common laboratory cell lines. Oligonucleotide primers homologous to conserved sequences flanking the protein coding region in Mus musculus and Rattus norvegicus were used to amplify RNase 6 from the above five rodent species. The primer sequences are as follows: forward, GAG CTA TGA AAT CAT AAA AGG AAG; and reverse, TTC CAA CAC CTT CCC TTT TCT GTG GGA ACA. DNA fragments of approximately 670 nucleotides were amplified from each of the five species. All PCR products were gel purified (BIO 101, Alta Vista, CA) and subcloned into pCR2.1 (Invitrogen, San Diego, CA) for automated DNA sequencing (ABI Prism, Applied Biosystems, Foster City, CA). The GenBank accession numbers are as follows: Mus musculus, AY545655; Rattus norvegicus, AY545654; Mus spicilegus, AY545653; Mus caroli, AY545651; and Mus pahari, AY 545652.

\section{Amplification of cDNA Ends (5' and $3^{\prime}$ RACE)}

To determine the precise transcriptional start and stop sites, rapid amplification of $5^{\prime}$ and $3^{\prime}$ cDNA ends was performed using liver and spleen Mus musculus mRNA (Clontech Laboratories, Inc., Palo Alto, CA). Complementary DNA was first synthesized from $1 \mu \mathrm{g}$ of total RNA with MMLV reverse transcriptase per the manufacturer's instructions (SMART RACE cDNA Amplification kit; Clontech) and then amplified using Mus musculus RNase 6 


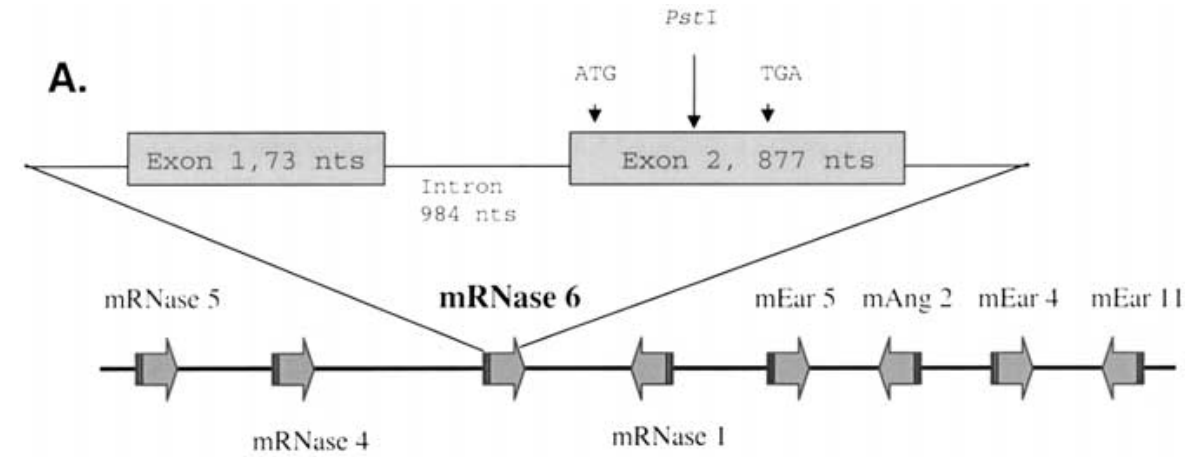

B.

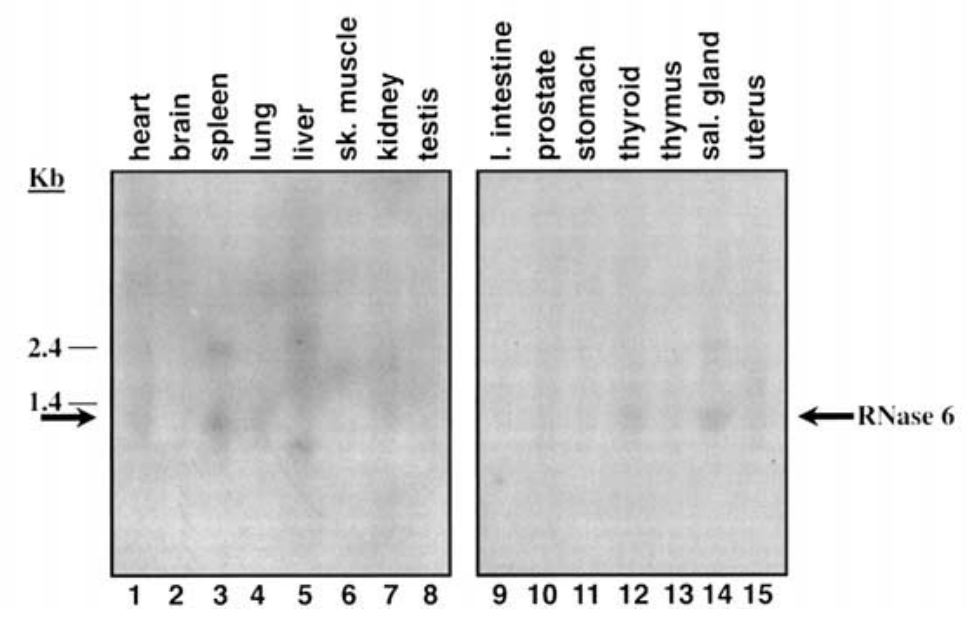

Fig. 2. Analysis of the Mus musculus RNase 6 expression and gene structure. A Gene structure and chromosomal localization of Mus musculus RNase 6. The gene structure is identical to that of other RNase A ribonucleases, with a single intron separating a noncoding and a coding exon. The gene is found on chromosome 14 and is flanked by RNase 4 and RNase 1. Members of the ribonuclease A superfamily were assigned location and directionality by BLAST alignment of the coding region against Build 30 of the mouse genome database as indicated by the arrows as previously reported by Dyer et al. (2004). B Expression in Mus musculus heart, spleen, thymus, and salivary gland. A 460-nucleotide probe inclusive of the start ATG and stop TGA was used to hybridize to commercially prepared Northern blots. The arrows mark the RNase 6 hybridization signal, which is consistent with the size deduced from sequencing the RACE products. The blots were also hybridized to actin to ascertain the RNA quality and relative concentration (data not shown). specific oligonucleotides (5' RACE, ACC GCG CAT GGC TGT GTT GCA TGG TTG ACG; 3' RACE, TCG TCA ACC ATG CAA CAC AGC CAT GCG CGG). The PCR conditions were as follows: 5 cycles at $94^{\circ} \mathrm{C}$ for $5 \mathrm{~s}$ followed by $72^{\circ} \mathrm{C}$ for $3 \mathrm{~min}$; then 5 cycles at $94^{\circ} \mathrm{C}$ for $5 \mathrm{~s}$ followed by $70^{\circ} \mathrm{C}$ for $10 \mathrm{~s}$ followed by $72^{\circ} \mathrm{C}$ for $3 \mathrm{~min}$; and finally, 25 cycles at $94^{\circ} \mathrm{C}$ for $5 \mathrm{~s}$ followed by $68^{\circ} \mathrm{C}$ for $10 \mathrm{~s}$ followed by $72^{\circ} \mathrm{C}$ for $3 \mathrm{~min}$. The cDNAs were gel purified (BIO 101) and subcloned into pCR2.1 (Invitrogen), and multiple colonies were sequenced in both directions. The sequences were assembled using Sequencher 4.1 (GeneCodes, Ann Arbor, MI) to obtain the full-length cDNA sequence.

\section{Northern and Southern Analyses}

Multiple-tissue Northern membranes containing mRNA from Mus musculus were purchased from Clontech. The membranes were prehybridized and hybridized following the manufacturer's instructions. The hybridization was performed with either a radiolabeled or a biotin-labeled (BrightStar psoralen-biotin; Ambion) open reading frame probe of Mus musculus RNase 6. The membranes were washed and exposed to film overnight. The actin gene was used as a control for RNA loading and integrity. For the Southern blot, $10 \mu \mathrm{g}$ of $129 \mathrm{SV}$ mouse genomic DNA provided by Jiliang Gao (National Institutes of Health, Bethesda, MD) was digested with the indicated restriction enzymes overnight at $37^{\circ} \mathrm{C}$. The digested DNA was electrophoresed on an $0.8 \%$ agarose gel and then transferred to a positively charged membrane. Following prehybridization, the blot was hybridized with the biotin-labeled probe.

\section{Generation of the Mus musculus RNase 6 Expression Construct and Production of Recombinant Protein}

The portion of the open reading frame encoding the predicted mature RNase 6 peptide (nucleotides 159-539; AY545655) was reamplified from liver cDNA using the following primers: forward, CTC CTC CTC AAG CTT CTC AGC CTA AGG GTC TCT CCA G; and reverse, CTC CTC CTC GAA TTC CAC AAT CTT ATC TAA GTG TA (underlined residues encode engineered restriction enzyme sites). The PCR product was subcloned into pCR2.1 for sequencing, and then the RNase 6 insert was excised using HindIII and EcoRI and ligated in frame into the prepared $\mathrm{pF}$ CTS bacterial expression vector (International Biotechnologies, Inc., New Haven, CT). The fidelity of the expression construct was confirmed by sequencing. Production and isolation of recombinant Mus musculus RNase 6 from bacterial transfectants were as described previously (Rosenberg et al. 1995). The concentration of recombinant protein was determined by comparison to serial dilutions of known concentrations of a FLAG-conjugated protein standard on Western blots. The Western blot was incubated with monoclonal anti-FLAG antibody (Sigma) followed by HPR-conjugated sheep anti-mouse $\operatorname{IgG}$ and developed with reagents from Amersham.

\section{Determination of Ribonuclease Activity Against tRNA Substrate}

The assay used was adapted from the procedure of Slifman et al. (1986) as described previously (Rosenberg et al. 1995). Calculations 
included the following approximations: the average molecular weight $\left(M_{r}\right)$ of tRNA as 28,100 (75-90 ribonucleotides/tRNA molecule $\times M_{r} 341 /$ ribonucleotide), with the $A_{260}$ of 1.0 corresponding to $40 \mu \mathrm{g}$ of RNA. All time points represent averages of triplicate samples. Equal volumes of vector-only protein isolations had levels of ribonuclease activity that were insignificant compared with that of recombinant Mus musculus RNase 6.

\section{Evolutionary Analysis of DNA Sequences}

Complete RNase 6 gene sequences from the five rodents, human, macaque, horse, and cow were aligned using ClustalX (Thompson et al. 1997). A neighbor-joining tree (Saitou and Nei 1987) was reconstructed with Kimura's (1980) distances. Bootstrap (Felsenstein 1985) percentages derived from 2000 replications were used to evaluate the reliability of the tree. Based on the reconstructed phylogeny, ancestral gene sequences at all interior nodes of the tree were statistically inferred using the distance-based Bayesian method (Zhang et al. 1997) and the numbers of synonymous and nonsynonymous substitutions on each branch of the tree were counted (Zhang et al. 1998). The number of synonymous nucleotide substitutions per synonymous site $\left(d_{\mathrm{S}}\right)$ and the number of nonsynonymous substitutions per nonsynonymous site $\left(d_{\mathrm{N}}\right)$ were computed using the method of Zhang et al. (1998).

\section{Results}

Discovery and Chromosomal Localization of Rodent RNase 6 Genes

Two overlapping sequences encoding Mus musculus RNase 6 were identified by homology to the human RNase 6 coding region (U64998) in the Celera Database. These sequences were used to identify the Mus musculus and Rattus norvegicus sequences in the public domain genome sequences. RNase 6 is located on Mus musculus chromosome 14 (NT_039599, Build 32) between RNase 4 and RNase 1 (Fig. 2A) and on chromosome 15 (NW_047453, Build 2) in Rattus norvegicus. These sequences were used for oligonucleotide design in order to amplify the Mus musculus gene and cDNA and Rattus norvegicus gene. Additionally, ESTs encoding RNase 6 from the cow Bos taurus (AF164025), macaque Macaca mulatta (CB554177), and horse Equus caballus (CD536566) were discovered by homology to the Mus musculus open reading frame through GenBank searches.

\section{Northern Analysis and Rapid Amplification of cDNA Ends ( $R A C E$ )}

RNase 6 transcripts of slightly less than $1 \mathrm{~kb}$ were detected in heart, spleen, thyroid, and salivary glands of mice (Fig. 2B). No hybridization signals of the correct size as determined by RACE (see below) were detected in the other tissues examined, suggesting that Mus musculus RNase 6 has a pattern of tissue expression that can be distinguished from that of human RNase 6 , which was readily detected by

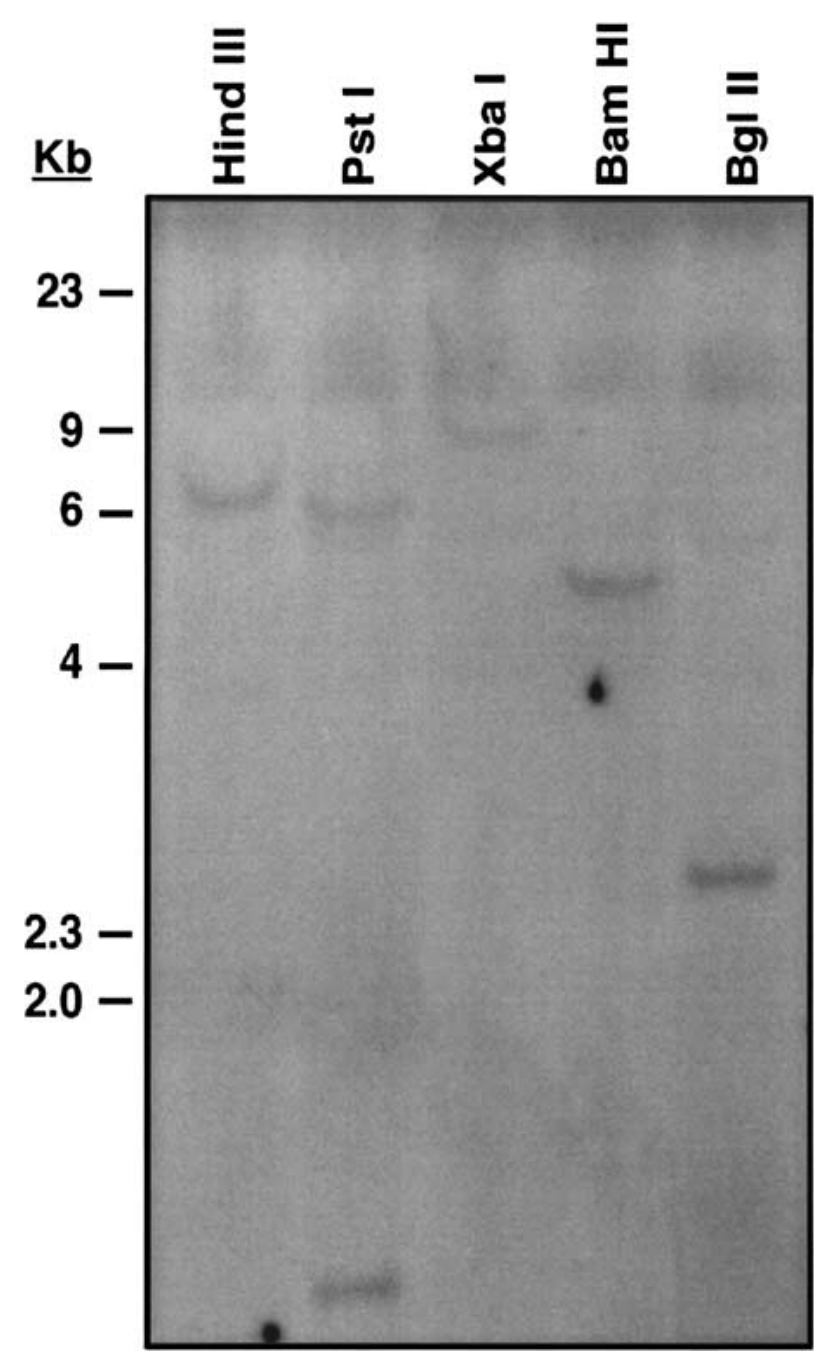

Fig. 3. Mus musculus RNase 6 is a single-copy gene. A 460nucleotide probe inclusive of the start ATG and stop TGA was used to hybridize to genomic DNA digested with HindIII, PstI, $X b a \mathrm{I}, B a m \mathrm{HI}$, and BglII. Of these enzymes, only Pst I cuts with the region homologous to the probe (see Fig. 2A).

Northern analysis in heart, brain, placenta, lung, liver, skeletal muscle, kidney, and pancreas (Rosenberg and Dyer 1996). Mus musculus RNase 6 transcripts were detected by RT-PCR in kidney, skeletal muscle, heart, bone marrow, and spleen (data not shown). Kidney and spleen RNAs were used to determine full transcript size. In addition to the experimentally derived cDNAs, several ESTs were found by homology search (AA182060, BE687377, BY722115, AK020595, BI964571, BI965795). However, these do not extend the cDNAs further, leading us to conclude that the full transcript size is 952 nucleotides. Gene structure was determined by mapping the complete cDNA onto the genomic fragment from GenBank (Fig. 2A) and is typical of the RNase A gene family, consisting of two exons - one noncoding exon 1 of 73 nucleotides and one coding exon 2 of 877 nucleotides - separated by an intron of 984 nucleotides. As mentioned above, Mus musculus RNase 6 is located 
Table 1. Percentage identity between predicted mature peptide sequences of RNase 6 genes: The sequences are as reported here and the mouse sequence is that of Mus musculus (AY545655)

\begin{tabular}{llllll}
\hline & Rat & Horse & Cow & Macaque & Human \\
\hline Mouse & 82 & 66 & 65 & 69 & 68 \\
Rat & - & 65 & 65 & 65 & 65 \\
Horse & - & - & 75 & 77 & 75 \\
Cow & - & - & - & 71 & 73 \\
Macaque & - & - & - & - & 94 \\
\hline
\end{tabular}

Lineweaver-Burke plot, also known as a double-reciprocal plot (Fig. 4A), was generated from 2.8 pmol of recombinant Mus musculus RNase 6, which was isolated from the periplasm of bacteria using the pFLAG CTS expression system (Fig. 4B). The values determined from the plot are $k_{\text {cat }}$, which refers to the number of molecules reacted per unit time; $K_{\mathrm{m}}$, which reflects the affinity of enzyme for the substrate; and $k_{\text {cat }} / K_{\mathrm{m}}$, which is a measure of the catalytic efficiency of the enzyme for the substrate. The catalytic constants for bacterial-derived Mus musculus RNase 6 are $k_{\text {cat }}=0.93 \mathrm{~s}^{-1}, K_{\mathrm{m}}=2.1 \mu \mathrm{M}$, and catalytic efficiency of $k_{\text {cat }} / K_{\mathrm{m}}=4 \times 10^{5} \mathrm{M}^{-1} \mathrm{~s}^{-1}$. The catalytic efficiency of Mus musculus RNase 6 is $\sim 17$-fold higher than that of human RNase $6\left[k_{\text {cat }}=0.13 \mathrm{~s}^{-1}\right.$, $K_{\mathrm{m}}=5 \mu \mathrm{M}, k_{\text {cat }} / K_{\mathrm{m}}=2.6 \times 10^{4} \mathrm{M}^{-1} \mathrm{~s}^{-1}$ (Rosenberg and Dyer 1996)]. The amino acid alignment of human and Mus musculus RNase 6 is shown in Fig. 4C. Human and mouse RNase 6 are $68 \%$ identical (Table 1) and each contains the eight cysteines necessary for folding, the histidines (amino acid positions 41 and 148) and lysine (position 64) that form the catalytic site, and the CKXXNTF motif found in all members of the RNase A superfamily.

\section{Evolutionary Analysis of RNase 6 in Multiple Species of Rodents}

To investigate further the evolution of RNase 6 among the rodent species, we isolated RNase orthologues by PCR amplification from genomic DNA using conserved sequence between Mus musculus (house mouse) and Rattus norvegicus (Norway rat). RNase 6 sequences were isolated from $M$. spiculegus (steppe mouse), M. caroli (Ryukyu mouse), and $M$. pahari (shrew mouse). Additional RNase 6 genes were identified by homology to Mus musculus RNase 6 in the EST database as mentioned above and these are from the following species: Bos taurus (cow), Macaca mulatta, (macaque monkey), and Equus caballus (horse). Table 1 shows the percentage identity between the predicted mature proteins. The rodent proteins are $81-96 \%$ identical, therefore $\mathrm{Mus} \mathbf{\text { muscu- }}$ lus-dervied RNase 6 was selected as representative of the rodent proteins and compared to those of non-
A.
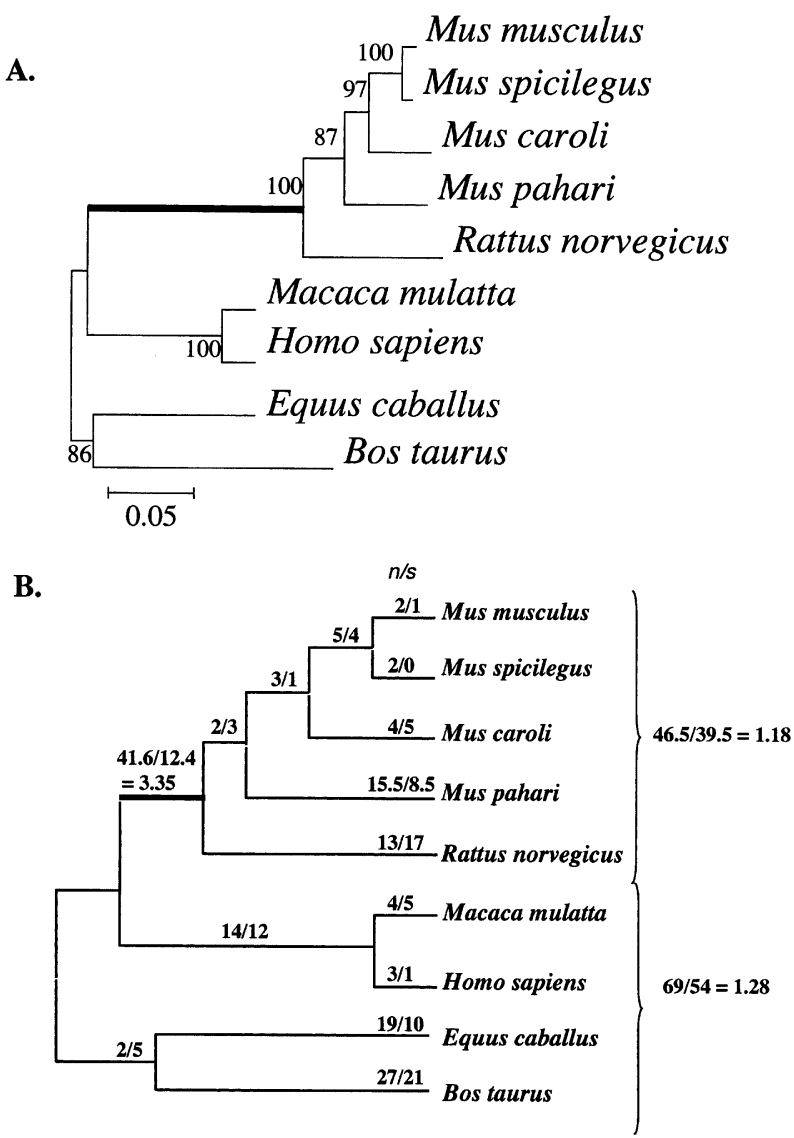

Fig. 5. Evolutionary analysis of the RNase 6 genes. A The neighbor-joining phylogenetic tree of the RNase 6 genes based on the DNA sequences of the coding region. The tree and bootstrap percentages were obtained using MEGA2 (Kumar et al. 2001). The ancestral rodent lineage discussed in the text is highlighted. Mus musculus, house mouse; Rattus norvegicus, Norway rat; M. spiculegus, steppe mouse; M. caroli Ryukyu mouse; M. pahari, shrew mouse; Bos taurus, cow; Homo sapiens, human; Macaca mulatta, macaque monkey; Equus caballus, horse. B Analysis of nonsynonymous versus synonymous changes in the RNase 6 lineages. Shown on each branch is the number of nonsynonymous substitutions $(n)$ divided by the number of synonymous substitutions $(s)$ that occurred in the branch. Then $n / s$ ratios are also computed for all the branches linking the five rodents and for all the branches linking the four nonrodent species.

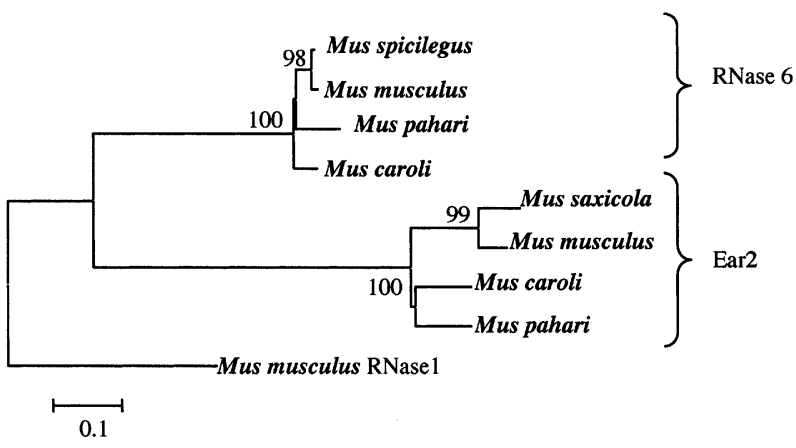

Fig. 6. Comparison of murine RNase 6 and Ear gene lineage based on the DNA sequences of the coding region. The tree and bootstrap percentages were obtained using MEGA2 (Kumar et al. 2001). The mouse RNase 1 gene (M27814) is used as the outgroup for this analysis. Mus musculus, house mouse; M. spiculegus, steppe mouse; $M$. caroli, Ryukyu mouse; M. pahari, shrew mouse, M. saxicola, spiny mouse. The RNase 6 gene sequences are as reported in this paper and the Ear sequences are as follows: AF238395, AF238402, AF238407, and U72031. 
Table 2. The murine RNase 6 gene is under purifying selection compared to the positive selection on the Ear 2 gene: The RNase 6 genes compared are those reported in this paper

\begin{tabular}{|c|c|c|c|}
\hline$d_{\mathrm{N}} / d_{\mathrm{S}}$ & Mus musculus & Mus caroli & Mus pahari \\
\hline Mus spicilegus (RNase 6) or Mus saxicola (Ear) & $1.39 / 0.97 *$ & $0.41 / 1.83$ & $0.58 / 1.11$ \\
\hline Mus musculus & - & $0.37 / 1.72$ & $0.52 / 1.21$ \\
\hline Mus caroli & - & - & $0.66 / 0.81$ \\
\hline
\end{tabular}

Note. The Ear GenBank accession numbers are as follows: Mus saxicola, AF238395; Mus musculus, U72031; Mus caroli, AF238407; Mus pahari, AF238402. The $d_{\mathrm{N}} / d_{\mathrm{S}}$ ratios were calculated using MEGA2 (Kumar et al. 2001).

Presented in each cell is the $d_{\mathrm{N}} / d_{\mathrm{S}}$ ratio for RNase 6 followed by that for Ear.

rodent species. The identity between Mus musculus RNase 6 and the nonrodent proteins averages $67 \%$ (Deming et al. 1998). The divergence of mouse and human RNase 6 is higher than what is generally found in mouse-human orthologues (Murphy 1993). The magnitude of divergence seen in the RNase 6 gene pair $(33 \%)$ is typical of the rapidly evolving genes involved in host defense (Murphy 1993).

The RNase 6 gene tree reconstructed with the neighbor-joining method recapitulated expected phylogeny, indicating that the sequences obtained are orthologous. Artiodactyls and Perissodactyls are thought to be outgroups of primates and rodents (Murphy et al. 2001; Thomas et al. 2003). Thus, the evolutionary rate of RNase 6 in rodents can be compared with that in primates using the horse and cow sequences as outgroups. This comparison reveals a substantially higher pace of evolution in rodents than in primates, as evident from the longer branches shown in Fig. 5A. This was not revealed in the initial gene tree (Fig. 1) due to the number of different members of the RNase A superfamily examined and the fact that this tree was constructed from protein sequences, which made the analysis between mouse and human RNase 6 less precise. The molecular clock hypothesis is rejected (Tajima's [1993] test, $p<1 \%$ ) when the rate of evolution is compared between any of the five rodents and either of the two primates in the tree. To investigate the accelerated evolution of RNase 6 in rodents, we inferred ancestral gene sequences at all interior nodes of the tree and counted the numbers of synonymous $(s)$ and nonsynonymous (n) substitutions for each tree branch (Fig. 5B). Since synonymous nucleotide changes are largely neutral and nonsynonymous changes may be subject to natural selection, a comparison between $s$ and $n$ can reveal the existence of natural selection on the gene. The average $n / s$ ratio for all the branches that link the five rodents is 1.18 . Similarly, the average ratio is 1.28 for all other branches of the tree except the bolded branch, which leads to the common ancestor of the five rodents investigated. For this ancestral rodent branch, the $n / s$ ratio is 3.35 , significantly higher than those in other branches of the tree $(p<0.01$, Fisher's test [Zhang et al. 1997]). This suggests that the rate of nonsynonymous substitutions relative to synonymous substitutions has been enhanced in RNase 6 during the early stages of rodent evolution. This may be caused by either a relaxation of functional constraints or positive Darwinian selection. To distinguish these two hypotheses, we compared the $n / s$ for this branch with the expected value $(N / S)$ when the gene is under neutral evolution. The $N / S$ value is estimated to be 2.79, using the method of Zhang et al. (1998). Thus $n / s$ is $\sim 1.2$ times $N / S$, but their difference is not statistically significant $(p>0.05)$, indicating that the sequence analysis alone cannot determine whether the accelerated evolution is due to relaxation of functional constraints or positive selection.

Modern murine RNase 6 and its closely related Ears (RNases 7 and 8 are absent from the mouse and rat genomes) are under different selective pressures as can be predicted by the different branch lengths shown in Fig. 6 . The $d_{\mathrm{N}} / d_{\mathrm{S}}$ ratios of mouse RNase 6 and Ear2 compared to those of human orthologues are not substantially different (0.58 and 0.49 , respectively), indicating a similar rate of evolution, however, the $d_{\mathrm{N}} / d_{\mathrm{S}}$ ratios among the gene lineages in different murine species are quite different (Table 2). The Ear2 gene lineage in the four murine species examined has an average $d_{\mathrm{N}} / d_{\mathrm{S}}$ of 1.28 , while the murine RNase 6 lineage has an average $d_{\mathrm{N}} / d_{\mathrm{S}}$ of 0.66 , indicating that the Ear family is evolving under positive selection as previously suggested (Zhang et al. 2000) while the related RNase 6 gene is more conserved.

\section{Discussion}

DNA sequences encoding segments of RNase 6 have been isolated from a variety of primate species (Deming et al. 1998; Rosenberg and Dyer 1996). Unlike the rapidly evolving eosinophil-associated ribonucleases, the RNase 6 gene appears to have evolved at a slow pace in primates (Deming et al. 1998). In this study, we report the DNA sequence and gene structure of Mus musculus RNase 6 and demonstrate that the expression pattern and enzymatic activity in this species is different from that of human RNase 6. In 
addition, we have sequenced the RNase 6 gene from Mus pahari, Mus caroli, Mus spicilegus, and Rattus norvegicus. Our analyses suggest that the RNase 6 gene was subject to altered evolutionary forces in the ancestral rodent lineage and that RNase 6 likely has altered functions in rodents.

By Northern analysis, mouse RNase 6 is found to be expressed in heart, spleen, and thyroid and salivary glands but undetectable in large intestine, prostate, stomach, thymus, uterus, brain, lung, skeletal muscle, or testis. Human RNase 6 has a much broader tissue expression profile and was readily detectable in all tissues examined including brain, lung, and skeletal muscle (Rosenberg and Dyer 1996). The transcript size of mouse RNase 6 is 952 nucleotides as determined by RACE and confirmed by Northern analysis, while the human transcript is slightly larger (1061 nucleotides) as determined by mapping ESTs against the human genome (unpublished data). In the mouse, the noncoding exon is 73 nucleotides, while that of the human RNase 6 gene is 288 base pairs, and the coding exon 2 is 879 and 773 base pairs, respectively. In addition to the difference in transcript size, the intron of the mouse gene is substantially longer than that found in the human gene (984 vs. 351 nucleotides). It has been shown for human EDN/RNase 2 (Tiffany et al. 1996), ECP/RNase 3 (Handen and Rosenberg 1997), and mouse Ear2 (Dyer et al. 2004) that the noncoding exon and intron contain important regulatory elements and perhaps these are disrupted or rearranged in the mouse RNase 6 gene, resulting in a more restricted expression profile compared to the human orthologue.

The comparison of the evolutionary rate of RNase 6 reveals a substantially higher rate of evolution in rodents than in primates. Analysis of the inferred ancestral gene sequences at all interior nodes of the RNase 6 tree showed that the ancestral rodent branch has an $n / s$ ratio of 3.35, significantly higher than the average $n / s$ for all other branches of the tree. This indicates that the rate of nonsynonymous relative to synonymous substitutions was enhanced in RNase 6 during the early stages of rodent evolution. Furthermore, the rate of nonsynonymous substitution in this branch is higher than the neutral expectation, although the difference is not statistically significant. This suggests that the accelerated evolution was due to either a complete relaxation of functional constraints or positive Darwinian selection. Because the important structural elements of RNase (eight cysteines and three catalytic residues) are all conserved in rodents, it is unlikely that the gene had no functional constraints during the early stage of rodent evolution. Given the altered expression profile and higher enzymatic activity for mouse RNase 6 , it seems more likely that nonsynonymous substitutions were promoted via the process of adaptive selec- tion. This is consistent with the observation that the $n / s$ ratio returned to a low level after the episode of rapid evolution, presumably because of the conservation of the altered function. This pattern of evolution is substantially different from that of the closely related Ear gene lineage. The mouse Ears continue to evolve at a much higher nonsynonymous substitution rate than the mouse RNase 6 gene lineage, with an average $d_{\mathrm{N}} / d_{\mathrm{S}}$ of 1.28 , compared to 0.66 for the RNase 6 lineage in equivalent species.

All these data suggest that the ancestral rodent RNase 6 gene was subject to accelerated evolution to some selective pressure that resulted in the conserved modern gene, which most likely has an important function in the mouse. A role in host defense would be consistent with the high rate of divergence between human and mouse and consistent with the diversity of function that is apparent among the members of the RNase A superfamily. The ultimate verification of this hypothesis, however, awaits the elucidation of the physiological functions of RNase 6 in rodents and other mammals.

Acknowledgment. Dr. Jianzhi Zhang is support by NIH Grant GM67030.

\section{References}

Batten D, Dyer KD, Domachowske JB, Rosenberg HF (1997) Molecular cloning of four novel murine ribonuclease genes: Unusual expansion within the ribonuclease A gene family. Nucleic Acids Res 25:4235-4239

Beintema JJ, Schuller C, Irie M, Carsana A (1988) Molecular evolution of the ribonuclease superfamily. Prog Biophys Mol Biol 51:165-192

Beintema JJ, Breukelman HJ, Dubois JY, Warmels HW (2003) Phylogeny of ruminants secretory ribonuclease gene sequences of pronghom (Antilocapra americana). Mol Phylogenet Evol $26: 18-25$

Cormier SA, Larson KA, Yuan S, Mitchell TL, Lindenberger K, Carrigan P, Lee NA, Lee JJ (2001) Mouse eosinophil-associated ribonucleases: A unique subfamily expressed during hematopoiesis. Mamm Genome 12:352-361

Deming MS, Dyer KD, Bankier AT, Piper MB, Dear PH, Rosenberg HF (1998) Ribonuclease k6: Chromosomal mapping and divergent rates of evolution within the RNase A gene superfamily. Genome Res 8:599-607

Domachowske JB, Bonville CA, Dyer KD, Rosenberg HF (1998a) Evolution of antiviral activity in the ribonuclease $\mathrm{A}$ gene superfamily: Evidence for a specific interaction between eosinophil-derived neurotoxm (EDN/RNase 2) and respiratory syncytial virus. Nucleic Acids Res 26:5327-5332

Domachowske JB, Dyer KD, Adams AG, Leto TL, Rosenberg HF (1998b) Eosinophil cationic protein/RNase 3 is another RNase A-family ribonuclease with direct antiviral activity. Nucleic Acids Res 26:3358-3363

Dubois JY, Ursing BM, Kolkman JA, Beintema JJ (2003) Molecular evolution of mammalian ribonucleases 1. Mol Phylogenet Evol 27:453-463

Dyer KD, Nitto T, Moreau JM, McDevitt AL, Rosenberg HF (2004) Identification of a purine-rich intronic enhancer element 
in the mouse eosinophil-associated ribnuclease 2 (mEar 2) gene. Mamm Genome 15:1-9

Felsenstein J (1985) Confidence limits on phylogenies: An approach using the bootstrap. Evolution 39:783-791

Futami J, Tsushima Y, Murato Y, Tada H, Sasaki J, Seno M, Yamada H (1997) Tissue-specific expression of pancreatic-type RNases and RNase inhibitor in humans. DNA Cell Biol 16:413-419

Handen JS, Rosenberg HF (1997) Intronic enhancer activity of the eosinophil-derived neurotoxin (RNS2) and eosinophil cationic protein (RNS3) genes is mediated by an NFAT-1 consensus binding sequence. J Biol Chem 272:1665-1669

Hooper LV, Stappenbeck TS, Hong CV, Gordon JI (2003) Angiogenins: A new class of microbicidal proteins involved in innate immunity. Nat Immunol 4:269-273

Kimura M (1980) A simple method for estimating evolutionary rates of base substitutions through comparative studies of nucleotide sequences. J Mol Evol 16:111-120

Kumar S, Tamura K, Jakobsen IB, Nei M (2001) MEGA2: Molecular evolutionary genetics analysis software. Bioinformatics 17:1244-1245

Lander ES, Linton LM, Birren B et al. (2001) Initial sequencing and analysis of the human genome. Nature 409:860-921

Larson KA, Olson EV, Madden BJ, Gleich GJ, Lee NA, Lee JJ (1996) Two highly homologous ribonuclease genes expressed in mouse eosinophils identify a larger subgroup of the mammalian ribonuclease superfamily. Proc Natl Acad Sci USA 93:1237012375

McDevitt AL, Deming MS, Rosenberg HF, Dyer KD (2001) Gene structure and enzymatic activity of mouse eosinophil-associated ribonuclease 2. Gene 267:23-30

Murphy PM (1993) Molecular mimicry and the generation of host defense protein diversity. Cell 72:823-826

Murphy WJ, Eizirik E, O'Brien SJ, Madsen O, Scally M, Douady CJ, Teeling E, Ryder OA, Stanhope MJ, de Jong WW, Springer MS (2001) Resolution of the early placental mammal radiation using Bayesian phylogenetics. Science 294:23482351

Pietrowski D, Forster M (2000) Complete cDNA sequence and amino acid analysis of a bovine ribonuclease K6 gene. DNA Seq 11:365-371

Rosenberg HF, Domachowske JB (2001) Eosinophils, eosinophil ribonucleases, and their role in host defense against respiratory virus pathogens. J Leukoc Biol 70:691-698

Rosenberg HF, Dyer KD (1995) Human ribonuclease 4 (RNase 4) Coding sequence, chromosomal localization and identification of two distinct transcripts in human somatic tissues. Nucleic Acids Res 23:4290-4295

Rosenberg HF, Dyer KD (1996) Molecular cloning and characterization of a novel human ribonuclease (RNase k6): Increasing diversity in the enlarging ribonuclease gene family. Nucleic Acids Res 24:3507-3513
Rosenberg HF, Dyer KD, Tiffany HL, Gonzalez M (1995) Rapid evolution of a unique family of primate ribonuclease genes. Nat Genet 10:219-232

Saitou N, Nei M (1987) The neighbor-joining method: A new method for reconstructing phylogenetic trees. Mol Biol Evol 4:406-425

Sasso MP, Lombardi M, Confalone E, Carsana A, Palmieri M, Furia A (1999) The differential pattern of tissue-specific expression of ruminant pancreatic type ribonucleases may help to understand the evolutionary history of their genes. Gene 227:205-212

Singhania NA, Dyer KD, Zhang J, Deming MS, Bonville CA, Domachowske JB, Rosenberg HP (1999) Rapid evolution of the ribonuclease A superfamily: Adaptive expansion of independent gene clusters in rats and mice. J Mol Evol 49:721-728

Slifman NR, Loegering DA, McKean DJ, Gleich GJ (1986) Ribonuclease activity associated with human eosinophil-derived neurotoxin and eosinophil cationic protein. J Immunol 137:2913-2917

Strydom DJ (1998) The angiogenins. Cell Mol Life Sci 54:811-824

Tajima F (1993) Simple methods for testing the molecular evolutionary clock hypothesis. Genetics 135:599-607

Thomas JW, Touchman JW, Blakesley RW et al. (2003) Comparative analyses of multi-species sequences from targeted genomic regions. Nature 424:788-793

Thompson JD, Gibson TJ, Plewniak F, Jeanmougin F, Higgins DG (1997) The CLUSTAL_X windows interface: Flexible strategies for multiple sequence alignment aided by quality analysis tools. Nucleic Acids Res 25:4876-4882

Tiffany HL, Handen JS, Rosenberg HF (1996) Enhanced expression of the eosinophil-derived neurotoxin ribonuclease (RNS2) gene requires interaction between the promoter and intron. J Biol Chem 271:12387-12393

Zhang J, Kumar S, Nei M (1997) Small-sample tests of episodic adaptive evolution: A case study of primate lysozymes. Mol Biol Evol 14:1335-1338

Zhang J, Rosenberg HF, Nei M (1998) Positive Darwinian selection after gene duplication in primate ribonuclease genes. Proc Natl Acad Sci USA 95:3708-3713

Zhang J, Dyer KD, Rosenberg HF (2000) Evolution of the rodent eosinophil-associated RNase gene family by rapid gene sorting and positive selection. Proc Natl Acad Sci USA 97: 4701-4706

Zhang J, Dyer KD, Rosenberg HF (2002a) RNase 8, a novel RNase A superfamily ribonuclease expressed uniquely in placenta. Nucleic Acids Res 30:1169-1175

Zhang J, Zhang YP, Rosenberg HF (2002b) Adaptive evolution of a duplicated pancreatic ribonuclease gene in a leaf-eating monkey. Nat Genet 30:411-415

Zhang J, Dyer KD, Rosenberg HF (2003) Human RNase 7: A new cationic ribonuclease of the RNase A superfamily. Nucleic Acids Res 31:602-607 